\title{
Normal Charge Densities in Quantum Critical Superfluids
}

\author{
Blaise Goutéraux $0^{*}$ and Eric Mefford $\odot^{\dagger}$ \\ CPHT, CNRS, École Polytechnique, IP Paris, F-91128 Palaiseau, France
}

(Received 13 January 2020; accepted 8 April 2020; published 22 April 2020)

\begin{abstract}
The normal density of a translation-invariant superfluid often vanishes at zero temperature, as is observed in superfluid Helium and conventional superconductors described by BCS theory. Here we show that this need not be the case. We investigate the normal density in models of quantum critical superfluids using gauge-gravity duality. Models with an emergent infrared Lorentz symmetry lead to a vanishing normal density. On the other hand, models which break the isotropy between time and space may enjoy a nonvanishing normal density, depending on the spectrum of irrelevant deformations around the underlying quantum critical ground state. Our results may shed light on recent measurements of the superfluid density and low energy spectral weight in superconducting overdoped cuprates.
\end{abstract}

DOI: 10.1103/PhysRevLett.124.161604

Introduction.-Much of traditional superfluid and BEC superconductor phenomenology can be explained by Landau and Tisza's simple two-fluid hydrodynamical model [1,2] and its relativistic generalizations [3-9]. The Landau-Tisza model describes the superfluid as a mixture of two fluid components, the normal, dissipative state with charge density $\rho_{n}$ and velocity $u_{\mu}$ and the dissipationless superfluid with charge density $\rho_{s}$ and flow velocity $v_{\mu}$. The total charge density is the sum of both components, $\rho=\rho_{n}+\rho_{s}$. Experiments and theoretical calcuations in ${ }^{3} \mathrm{He},{ }^{4} \mathrm{He}$, cold atom experiments, and conventional BCS superconductors all lead to the result that the system becomes entirely superfluid at zero temperature, i.e., that the normal density vanishes:

$$
\rho_{n}^{(0)} \equiv \lim _{T \rightarrow 0} \rho_{n}=0 .
$$

In $[10,11]$, two arguments were given to account for (1). One argument used only the superfluid hydrodynamic description, the other assumed a weakly coupled, Galilean, time-reversal invariant, single species superfluid.

The expectation (1) was called into question by recent experimental reports of anomalously low superfluid densities in overdoped high- $T_{c}$ superconductors [12] (see $[13,14]$ for commentary). Subsequent spectroscopic studies [15] also revealed very little loss of low energy spectral weight at low temperatures in the superconducting phase, suggesting a nonvanishing $\rho_{n}^{(0)}$. While the authors of [16-18] attributed this to disorder effects that can be

Published by the American Physical Society under the terms of the Creative Commons Attribution 4.0 International license. Further distribution of this work must maintain attribution to the author(s) and the published article's title, journal citation, and DOI. Funded by SCOAP ${ }^{3}$. captured in the so-called dirty BCS theory, fitting the experimental data relies on an ad hoc renormalization of the Drude weight [17]. Thus, no theoretical consensus has been reached on the experimental findings of $[12,15]$, see also $[19,20]$.

In this Letter, we tackle this question by combining methods using superfluid hydrodynamics and gaugegravity duality. We review translation and time-reversal invariant superfluid hydrodynamics and show that the hydrodynamic equations are not enough to conclude that (1) is true. Instead, determining $\rho_{n}^{(0)}$ requires knowledge of the infrared (IR) equation of state. Using holographic models with quantum critical dynamics in the infrared as examples, we show (1) holds for strongly coupled superfluids with an emergent Lorentz symmetry, in agreement with $[10,11]$. This is also consistent with the superfluid effective field theory discussed in [21-23]. On the other hand, we find that nonrelativistic quantum critical systems with dynamical critical exponent $z>1$ can have $\rho_{n}^{(0)} \neq 0$. Hence, we conclude that a nonvanishing $\rho_{n}^{(0)}$ is not a result of the breakdown of the two-fluid model but rather a result of the quantum critical nature of the IR of these superfluids. Even after explicitly breaking translations, we show this conclusion does not change. These findings may suggest the anomalously low superfluid density and suppression of spectral weight observed in $[12,15]$ might be a consequence of the quantum critical properties of the superconducting phase of overdoped cuprates.

On a more formal level, our results indicate that the quantum effective action of Lifshitz superfluids differs significantly from that of their Lorentzian cousins [21], which opens exciting perspectives for future research on the theory of superfluids.

$\rho_{n}^{(0)}$ in superfluid hydrodynamics. - In this section, we review relativistic, charged, superfluid hydrodynamics, 
following [24]. For our purposes, it is sufficient to work at the nondissipative level. Our results apply to any theory with translation invariance, including Galilean invariant theories. Relativistic symmetry leads to simpler notation and aligns nicely with our holographic example. A more thorough derivation can be found in [25].

The system is described by the following equations (setting the speed of light $c=1$ )

$$
\begin{aligned}
\partial_{\mu} T^{\mu \nu} & =0, & & \partial_{\mu} j^{\mu}=0, \\
u^{\mu} \partial_{\mu} \varphi+\mu & =0, & & \partial_{\mu}\left(s u^{\mu}\right)=0 .
\end{aligned}
$$

The first line expresses the local conservation laws: the conservation of the fluid stress tensor and the conservation of the U(1) symmetry current, respectively. The last line states the constraints from gauge invariance and thermodynamics: respectively, a "Josephson equation" which relates the time component of the background gauge field to the phase of the superfluid, $\varphi$, and the statement that in equilibrium, the entropy density is conserved. For simplicity, we have turned off external sources, which in particular corresponds to a choice of gauge $A_{\mu}=0$ for the external gauge field. The conclusions of this Letter are independent of the choice of gauge, see [25].

Hydrodynamics states that these equations can be solved in terms of a derivative expansion of local thermodynamic variables and the fluid velocity. At nondissipative order, thermodynamics of the equilibrium state fixes

$$
\begin{aligned}
T^{\mu \nu} & =\left(\epsilon_{n}+P\right) u^{\mu} u^{\nu}+P \eta^{\mu \nu}+\frac{\rho_{s}}{\mu} \partial^{\mu} \varphi \partial^{\nu} \varphi, \\
j^{\mu} & =\rho_{n} u^{\mu}+\frac{\rho_{s}}{\mu} \partial^{\mu} \varphi .
\end{aligned}
$$

The total charge density is the sum of the normal, $\rho_{n}$, and superfluid, $\rho_{s}$, densities. The distinction between $\rho_{n}$ and $\rho_{s}$ follows from the expectation that $u^{\mu}$ is the velocity of entropy flow which is carried purely by the normal component. The normal energy density, $\epsilon_{n}$, and pressure, $P$, satisfy the Smarr and Gibbs relations,

$$
\begin{aligned}
\epsilon_{n}+P & =T s+\rho_{n} \mu, \\
d P & =s d T+\rho d \mu-\frac{\rho_{s}}{2 \mu} d\left(\partial_{\nu} \varphi \partial^{\nu} \varphi+\mu^{2}\right) .
\end{aligned}
$$

We perturb about equilibrium, writing $T=T_{0}+\delta T$, $\mu=\mu_{0}+\delta \mu, u^{\mu}=\left(1, \delta u^{i}\right)^{\mu}, \varphi=-\mu_{0} t+\delta \varphi$. The fluctuation equations can be massaged into the form

$0=\left(\mu \rho_{n}+s T\right) \partial_{t} \delta u_{i}+\rho_{s} \partial_{t} \partial_{i} \delta \varphi+s \partial_{i} T+\rho \partial_{i} \delta \mu$.

If $s \rightarrow 0$ as $T \rightarrow 0$, consistency of this equation requires

$$
\rho_{n}^{(0)} \partial_{t}\left(\mu \delta u_{i}-\partial_{i} \delta \varphi\right)=0 .
$$

If $\delta u_{i}$ and $\delta \varphi$ were allowed to fluctuate independently, we would conclude $\rho_{n}^{(0)}=0$, as in [10,11].
However, introducing an external source for $\varphi$ through $\delta H=\int d^{2} x s_{\varphi} \partial \varphi$ leads to $s_{\varphi}^{i}=\rho_{s}\left(\partial^{i} \varphi-\mu u^{i}\right) \quad[25,26]$. Setting the external source to zero, the superfluid velocity $v^{i} \equiv \partial_{i} \varphi / \mu$ is aligned with the fluid velocity $u^{i}$ and equation (6) is automatically satisfied. Therefore, consistent coupling of the hydrodynamics to external sources evades the conclusion that $\rho_{n}^{(0)}=0$.

The fluctuations lead to an electrical conductivity at nondissipative order [35],

$$
\sigma(\omega)=\frac{i}{\omega} G_{J^{x} J^{x}}^{R}(\omega, 0)=\frac{i}{\omega}\left[\frac{\rho_{n}^{2}}{\mu \rho_{n}+s T}+\frac{\rho_{s}}{\mu}\right] .
$$

In general, contact terms may affect the conductivities [36], but this is not the case for the electric conductivity of interest here. Importantly, $\lim _{T \rightarrow 0} \omega \operatorname{Im}[\sigma]=\rho / \mu$, irrespective of whether $\rho_{n}^{(0)}=0$ or not. Here, as well as everywhere in the rest of our Letter, we take the $\omega \rightarrow 0$ limit before the $T \rightarrow 0$ limit. The Kramers-Kronig relations require that $\operatorname{Re}[\sigma]$ also has a delta function as $\omega \rightarrow 0$ with the same weight. Equation (7) applies equally well to superconductors with a dynamical gauge field, as the conductivity is measured with respect to the total electric field, which relates it to the unscreened retarded Green's function.

If we explicitly break translations weakly, the momentum relaxes slowly with an inverse lifetime $\Gamma$ and the conductivity becomes

$$
\sigma(\omega)=\frac{\rho_{n}^{2}}{\mu \rho_{n}+s T} \frac{1}{\Gamma-i \omega}+\frac{\rho_{s}}{\mu}\left(\frac{i}{\omega}\right) .
$$

The imaginary pole is now proportional only to the superfluid density, though this says nothing about $\rho_{n}^{(0)}$. Importantly, there are no inconsistencies if $\rho_{n}^{(0)}=0$ when translations are broken, as we will demonstrate.

Holographic quantum critical superfluids.-Holography relates the low energy dynamics of a finite temperature strongly interacting gauge theory with a large number of colors in $d+1$ spacetime dimensions to the dynamics of a classical gravitational system in $d+2$ dimensions with a black hole [37,38]. While explicit examples are known from string theory that fix the action of the gravitational theory, applied holography posits that a consistent set of a small number of fields, such as scalars and $\mathrm{U}(1)$ gauge fields, coupled to gravity in $(d+2)$ anti-de Sitter spacetime is able to capture the universal low energy dynamics of a large number of strongly interacting quantum systems near a quantum critical point or phase [39].

In particular, these quantum critical theories should be characterized by the dependence of correlation functions on certain universal exponents, for instance the dynamical critical exponent, $z$, the hyperscaling violation parameter $\theta$, and the spatial dimension $d$. Holographically, these exponents are captured by an extremal (zero temperature) horizon of the form $[40,41]$ 


$$
d s^{2}=r^{2(\theta / d)-2}\left[-L_{t}^{2} \frac{d t^{2}}{r^{2 z-2}}+\tilde{L}^{2} d r^{2}+L_{x}^{2} d x^{2}\right]
$$

where the horizon is at $r \rightarrow \infty$ when $z \geq 1$. The radial coordinate $r$ functions as a renormalization scale so that under scale transformations,

$$
\left(r, x^{i}\right) \rightarrow \lambda\left(r, x^{i}\right) t \rightarrow \lambda^{z} t \Rightarrow d s^{2} \rightarrow \lambda^{2 \frac{\theta}{d}} d s^{2} .
$$

This implies that the thermodynamic parameters have dimension $[T]=z$ and $[s]=d-\theta$ and $s \sim T^{(d-\theta) / z}$.

A very general gravitational model that can lead to these extremal solutions is the following [42],

$$
\begin{aligned}
S= & \int d^{d+2} x \sqrt{-g}\left[R-\frac{Z(\phi)}{4} F^{2}-|D \eta|^{2}-\frac{1}{2}(\partial \phi)^{2}\right. \\
& \left.-V(\phi,|\eta|)-\frac{Y(\phi)}{2} \sum_{i=1}^{d}\left(\partial \psi_{i}\right)^{2}\right] .
\end{aligned}
$$

Here, $A_{M}$ is a U(1) gauge field with field strength $F_{M N}=$ $\partial_{M} A_{N}-\partial_{N} A_{M}$. The field $\eta$ is a complex scalar with $\mathrm{U}(1)$ charge $Q$ and covariant derivative $D \eta=\left(\partial_{M}-i Q A_{M}\right) \eta$. When $|\eta| \neq 0$, the $\mathrm{U}(1)$ symmetry is broken and the dual theory can be thought of as a superfluid $[27,43]$. The field $\phi$ is a neutral scalar called the dilaton which has a source on the boundary $\phi_{s}$. The fields $\psi_{I}$ are chosen to have linear dependence on the spatial dimensions, $\psi_{i}=m x^{j} \delta_{i j}$ so that when $Y \neq 0$, they explicitly break translation but not rotation invariance [28]. The gauge field is chosen only to have a background time component whose value at the boundary of anti-de Sitter sets the chemical potential, $\mu$, which sources a charge density, $\rho$. We have set $16 \pi G=1$. See [25] for further details.

The relativistic invariant equations of superfluidity described above were shown to hold in holographic models where the transport coefficients can be derived from the gravitational dual to the boundary fluid [24,29,44-47]. Though the early holographic models focused on the original holographic superfluid $[27,43,48]$, the bulk action can be generalized as in (11) to include running couplings and bounded scalar potentials [42,49-51]. We find that the two-fluid hydrodynamic model still works well in describing these models.

The solutions (9) are found for potentials which behave in the IR as

$$
\begin{aligned}
Y(\phi \rightarrow \infty) & \rightarrow Y_{0} e^{\lambda \phi}, & V(\phi,|\eta|) \rightarrow V_{0} e^{-\delta \phi}, \\
Z(\phi \rightarrow \infty) \rightarrow Z_{0} e^{\gamma \phi}, & & \phi=\kappa \ln (r) .
\end{aligned}
$$

The gauge field and translation breaking scalars can be engineered to be marginal or irrelevant deformations of the IR critical phase [41,52]. We will be concerned with phases where the charged scalar is irrelevant in the IR, taking the asymptotic value $\eta_{0}$ [42]. This implies that the scaling exponents are the same in the superfluid as in the normal phase, so that many of the scaling properties at low temperature are inherited from the normal phase.

Normal densities in holographic superfluids.-To find the normal density (see also [44]), we perturb our system by turning on a spatially homogeneous infinitesimal external electric field in the $x$ direction, $E_{x} e^{-i \omega t}$, sourcing both an electric and a momentum current [25]. As $\omega \rightarrow 0$, the equation for the momentum current enforces $\left\langle T_{t x}\right\rangle=-\rho \delta \xi_{x}$, where $\delta \xi_{x}=\partial_{x} \varphi-A_{x}$ is gauge invariant, and given by the electric field $\delta \xi_{x}=E_{x} /(i \omega)$ in a gauge where $\varphi=0$, which is the gauge we work in. This response requires that $\mu \delta u_{x}=\delta \xi_{x}[25]$.

In the companion paper [53], we explore transport in the superfluid phases of the holographic model (11) for general potentials in greater detail. Here, for illustrative purposes, we present an explicit example in $d=2$ that leads to $\rho_{n}^{(0)} \neq 0$ and one that leads to $\rho_{n}^{(0)}=0$, including when translations are broken. Specifically, we use the model of [42] with

$Z=e^{\phi / \sqrt{3}}, \quad V=-6 \cosh (\phi / \sqrt{3})-2|\eta|^{2}+|\eta|^{4}, \quad Y=0$.

Upon varying the dilaton source, this model has two IR phases characterized by critical exponents,

$$
\left(z, \theta, \frac{z}{\theta}\right)=(+\infty,-\infty,-1), \text { or }(z, \theta)=(1,-1) .
$$

In the first case, we first need to redefine $r \mapsto r^{1 / z}$ before sending $z \rightarrow+\infty$ in (9). The IR behavior of $\phi$ in the two phases is $\phi= \pm \sqrt{3} \ln (r)$. In the first case, $Z(\phi)$ diverges and leads to a finite electric flux, $\rho_{\text {in }}^{(0)}$, from the extremal horizon, suggesting a "fractionalization" of charged degrees of freedom into a subset confined in the condensate and subset deconfined in the thermal bath hidden by the horizon [54,55]. In the second case, $Z(\phi) \rightarrow 0$ causing the flux to vanish in the IR and all charged degrees of freedom are confined into the charged condensate in a "cohesive" phase.

These two cases can also be distinguished by the vanishing of $\rho_{n}^{(0)}$ in the cohesive phase and nonvanishing of $\rho_{n}^{(0)}$ in the fractionalized phase. We emphasize that, despite their apparent similarities, $\rho_{n}^{(0)}$ and $\rho_{\text {in }}^{(0)}$ are not immediately related. The first is a quantity defined in the two-fluid hydrodynamic model while the second is a microscopic measurement of the uncondensed degrees of freedom. This is analogous to BEC superconductivity where not all electrons condense into Cooper pairs, yet $\rho_{n} \rightarrow 0$ [56]. In fact, in [53], we discuss pure Lifshitz superfluid solutions $[49,50]$ in which $\rho_{\text {in }}^{(0)}$ vanishes while $\rho_{n}^{(0)}$ does not, for sufficiently large $z$. 

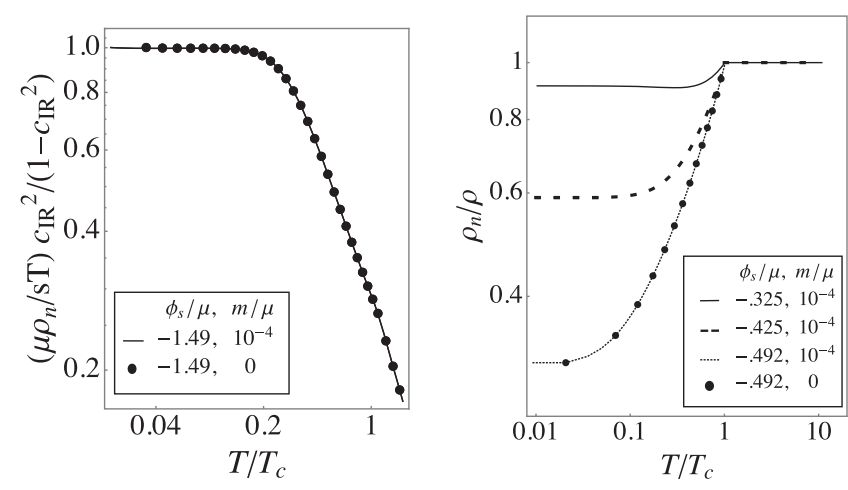

FIG. 1. Temperature dependence of $\rho_{n} / \rho$ in the cohesive (left) and fractionalized (right) phases.

After solving for the bulk $\delta \xi_{x}(r)$, we combine (7) with the knowledge of the total background charge density $\rho=$ $\rho_{s}+\rho_{n}$ to extract both $\rho_{n}$ and $\rho_{s}$. Our numerical results are shown in Fig. 1. In [53], we show analytically that

$$
\begin{gathered}
\text { fractionalized: } \rho_{n} \simeq \frac{\rho_{n}^{(0)}}{\mu^{2}}+\# T^{1-\frac{\theta}{z}}+, \cdots, \\
\text { cohesive }: \rho_{n} \simeq \frac{1-c_{\mathrm{IR}}^{2}}{c_{\mathrm{IR}}^{2}} \frac{s T}{\mu}+, \cdots \sim T^{(2+z-\theta) / z},
\end{gathered}
$$

where $\rho_{n}^{(0)}$ depends on UV parameters, for instance, the source, $\phi_{s}$, and $c_{\mathrm{IR}} \equiv L_{t} / L_{x} r_{h}^{1-z} \sim T^{1-1 / z}$ is the light cone velocity in the IR. The ... indicate terms from more irrelevant deformations of the IR geometry. Interestingly, the leading order temperature dependencies of the normal density behave as power laws with exponents determined by the underlying IR phase, characteristic of quantum critical systems. This is in contrast to BCS superconductivity, in which it is found that $\rho_{n}$ is exponentially suppressed [10]. On the other hand, in ${ }^{4} \mathrm{He}$, the normal (mass) density is controlled by phonons [the goldstones from the U(1) breaking] so that $\rho_{n}=(s T) / c_{p}^{2}+O\left(c_{p}^{-1}\right)$ where the coefficient is the phonon speed of sound, $c_{p}$ [57]. This is identical to (16), trading $c_{\mathrm{IR}} \mapsto c_{p}$ and taking the limit $c_{p} \ll 1$.

In [53], we find that $\rho_{n}^{(0)}$ depends on the competition between two terms proportional to $s T$ and $c_{\mathrm{IR}}^{2}$, respectively [58]. If $c_{\mathrm{IR}}^{2}$ dominates at low $T$, then $\rho_{n}^{(0)} \neq 0$. Otherwise, $\rho_{n}^{(0)}=0$ and to leading order $\rho_{n}$ is given by (16). This result is consistent with the relativistic superfluid effective field theory [23], but is also true for $z \neq 1$. For the quantum critical superfluids presented here, fractionalized phases $\left(\rho_{\text {in }}^{(0)} \neq 0\right)$ always have $c_{\mathrm{IR}}^{2}>s T$ and hence $\rho_{n}^{(0)} \neq 0$, whereas for cohesive phases $\left(\rho_{\text {in }}^{(0)}=0\right)$, this occurs for:

$$
\text { cohesive: } z<d+2-\theta \Rightarrow \rho_{n}^{(0)}=0 \text {. }
$$

We observe that when (17) is violated, (16) would naïvely lead to a divergent $\rho_{n}^{(0)}$. Instead, as we have just explained, a more careful calculation leads to a finite $\rho_{n}^{(0)} \neq 0$.
Generically, many irrelevant deformations of the critical IR geometry compete to drive the system toward the UV. In particular, while a universal deformation proportional to $s T$ always exists, dangerously irrelevant operators may control the temperature dependence of thermodynamic or transport observables $[41,59,60]$. It is then remarkable that the criteria in (17) leads to the universal temperature dependence (16) for cohesive phases.

As a final illustration that $\rho_{n}^{(0)} \neq 0$ is a signature of criticality rather than, for instance, disorder, we explicitly break translations in $(11)$, with $Y(\phi)=\exp (\mp \phi / \sqrt{3})$, where the minus sign is for fractionalized phases and the plus for cohesive phases. This choice ensures that translation breaking is sufficiently irrelevant to not destabilize the IR geometry. We omit the detailed accounting of gauge invariant fluctuations which can be found, for instance, in [61]. Due to the introduction of broken translations, we confirm $\lim _{\omega \rightarrow 0} \omega \operatorname{Im}[\sigma]=\rho_{s} / \mu$ (see also [62-64]) as in (8) and find $\Gamma=m^{2} s Y\left(r_{h}\right) /\left(4 \pi\left[\mu \rho_{n}+s T\right]\right)$ as follows from [41], see Fig. 2. Furthermore, the temperature dependence in Eqs. (15) and (16) does not change. In particular, translation symmetry breaking does not necessarily give rise to finite $\rho_{n}^{(0)}$ in the cohesive phase. Instead, $\rho_{n}^{(0)}$ is controlled by the underlying criticality.

Low temperature behavior of hydrodynamic modes.Equation (16) has interesting consequences on the spectrum of hydrodynamic modes at low temperatures. The superfluid second sound velocity is given by $[29,44]$

$$
c_{2}^{2}=\left(\frac{s}{\rho}\right)^{2} \frac{\rho_{s}}{\left(s T+\mu \rho_{n}\right)(\partial[s / \rho] / \partial T)_{\mu}} .
$$

Using (16) and $s \sim T^{(d-\theta) / z}$, we find $c_{2}^{2}=z c_{\mathrm{IR}}^{2} /(d-\theta)$. This is the generalization of Landau's conjecture [65] to critical IR geometries. For fractionalized phases, on the other hand, we find $c_{2}^{2} \sim s T$, which decays parametrically faster with temperature than $c_{\mathrm{IR}}$ when (17) holds. In both cases, the superfluid sound velocity vanishes at $T=0$. This is in marked contrast to the relativistic case $z=1$ and the
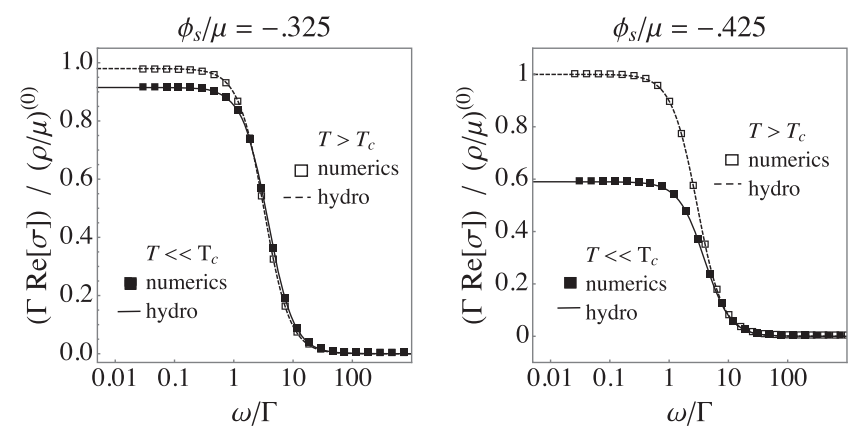

FIG. 2. Varying depletion of spectral weight in the real part of $\sigma(\omega)$ due to the superfluid in the presence of broken translations $\left(m / \mu=10^{-2}\right)$. Lower depletion is correlated with a larger $\rho_{n}^{(0)}$. The curves are the real part of (8). 
superfluid effective field theory [23], which lead to a nonvanishing $T=0$ superfluid velocity. We expect the Goldstone mode should interpolate to a dispersion relation $\omega \sim k^{z}$ in the limit $T \ll k$. It would be interesting to work this out in our model.

Fourth sound is defined as the sound mode which propagates when the normal velocity vanishes [65], given by

$$
c_{4}^{2}=\frac{\rho_{s}}{\mu\left(\frac{\partial \rho}{\partial \mu}\right)_{s}} \simeq \frac{\rho_{s}}{d \rho} .
$$

In the second equality, we have used the low temperature behavior, $\rho \sim \mu^{d}$. Thus, the fourth sound provides a direct measure of whether $\rho_{n}^{(0)}=0$, since then $c_{4}^{2}=1 / d$. This result explains some observations reported in previous literature $[44,66]$. In dirty superfluids with broken translations, only the fourth sound survives. In particular, (19) matches the expressions in [67]. Thus, measuring superfluid sound in impure, quantum critical superfluids would give direct information on whether $\rho_{n}^{(0)}=0$ or not.

Discussion.-In this Letter, we have shown that a nonvanishing $\rho_{n}^{(0)}$ is consistent with the Landau-Tisza two-fluid model of superfluidity. This is because in the absence of external sources, fluctuations in the normal velocity are aligned with fluctuations in the superfluid velocity, $\mu \delta u_{i}=\partial_{i} \delta \varphi$. We illustrated this using a model of holographic superfluidity and showed that $\rho_{n}^{(0)}$ is controlled by the underlying quantum critical phase. Experimental evidence for a nonvanishing $\rho_{n}^{(0)}$ in the cuprates can be considered further evidence for the existence of an underlying quantum critical phase in those systems. It would be interesting to find more experimental examples of nonvanishing $\rho_{n}^{(0)}$, perhaps in cold atom experiments, which we expect would be a generic feature of quantum criticality.

The holographic models discussed here exhibit further similarities to experimental observations in the cuprates. In overdoped $\mathrm{La}_{2-x} \mathrm{Sr}_{x} \mathrm{CuO}_{4}$, [68,69], heat capacity measurements reveal a linear in temperature component at low temperatures, $c \sim \gamma_{0} T+O\left(T^{2}\right)$. The coefficient, $\gamma_{0}$, is a measure of the density of normal charge carriers and exhibits strong doping dependence that is correlated with strong depletion of spectral weight in the optical conductivity $[12,15]$. Interpreting a source for the dilaton, $\phi_{s}$, as a proxy for doping [70], our models exhibit the same behavior, illustrated in Figs. 2 and 3. The rapid depletion arises from the underlying quantum critical point separating the cohesive phase in which $\gamma_{0}=0$ and the fractionalized phase in which $\gamma_{0} \neq 0$. As we have illustrated, this phase transition also separates phases in which $\rho_{n}^{(0)}$ does and does not vanish. Together, these observations give further evidence that a transition between two types of quantum critical phases may explain the phenomenology in the overdoped cuprates, see also [71-73].
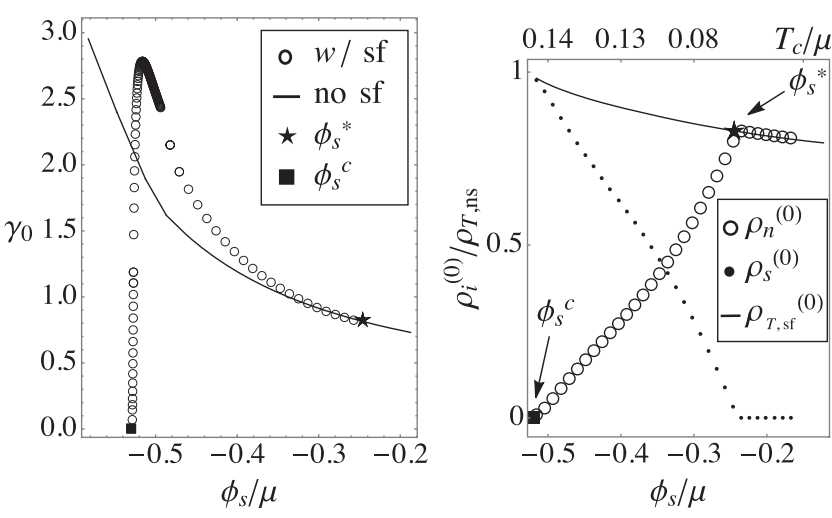

FIG. 3. Left: the linear specific heat slope, $\gamma_{0}$, as a function of the dilaton source, $\phi_{s}$, mimicking Fig. (5) of [68]. Right: the zero temperature densities as a function of $\phi_{s}$ compared to the total density with no superfluid, $\rho_{T, n s}$ mimicking Fig. (2e) of [15]. Two special points are indicated: $\phi_{s}^{c}$ marks the phase transition between cohesive and fractionalized phases and $\phi_{s}^{*}$ marks the phase transition to a totally fractionalized phase with superfluidity completely suppressed, $\rho_{s}^{(0)}=0$.

As a final remark, we observe that in a Lifshitz quantum critical fractionalized phase, our result (15) implies $\rho_{s} \simeq \rho_{s}^{(0)}+\# T^{1-\theta / z}$. Setting $\theta=0$, the superfluid density displays a universal $T$-linear scaling for all $z>1$. A similar observation was reported by recent experiments in overdoped $\mathrm{La}_{2-x} \mathrm{Sr}_{x} \mathrm{CuO}_{4}$ [12]. For $z=2$, the heat capacity will also receive a $T$-linear contribution. The value $z=2$ has appeared previously in theoretical models of high $T_{c}$ superconductors, see, e.g., [74-76].

We would like to thank Nigel Hussey, Catherine Pepin, and Steve Kivelson for useful discussions. In addition, we would like to thank Tomas Andrade and Richard Davison for initial collaboration at an early stage of this project. We would also like to thank Luca Delacrétaz for many discussions on superfluid hydrodynamics. We are grateful to Richard Davison, Sean Hartnoll, Chris Herzog, and Jan Zaanen for helpful comments on a previous version of this manuscript. We would especially like to thank Jan Zaanen who first brought the results of [12] to our attention at the Aspen Center for Physics, where this work was initiated and which is supported by National Science Foundation Grant No. PHY-1607611. This work was supported by the European Research Council (ERC) under the European Union's Horizon 2020 research and innovation programme (Grant Agreement No. 758759).

*blaise.gouteraux@polytechnique.edu †eric.mefford@polytechnique.edu

[1] L. Landau, Theory of the superfluidity of helium ii, Phys. Rev. 60, 356 (1941).

[2] L. Tisza, The theory of liquid helium, Phys. Rev. 72, 838 (1947). 
[3] I. M. Khalatnikov and V. V. Lebedev, Relativistic hydrodynamics of a superfluid liquid, Phys. Lett. 91A, 70 (1982).

[4] V. V. Lebedev and I. M. Khalatnikov, Relativistic hydrodynamics of a superfluid liquid, Zh. Eksp. Teor. Fiz. 56, 1601 (1982).

[5] B. Carter and I. M. Khalatnikov, Equivalence of convective and potential variational derivations of covariant superfluid dynamics, Phys. Rev. D 45, 4536 (1992).

[6] B. Carter and I. M. Khalatnikov, Momentum, vorticity, and helicity in covariant superfluid dynamics, Ann. Phys. (N.Y.) 219, 243 (1992).

[7] W. Israel, Covariant superfluid mechanics, Phys. Lett. 86A, 79 (1981).

[8] W. Israel, Equivalence of two theories of relativistic superfluid mechanics, Phys. Lett. 92A, 77 (1982).

[9] B. Carter and D. Langlois, The Equation of state for cool relativistic two constituent superfluid dynamics, Phys. Rev. D 51, 5855 (1995).

[10] A. James Leggett, Quantum Liquids: Bose Condensation and Cooper Pairing in Condensed-Matter Systems (Oxford University Press, Oxford, 2006).

[11] A. J. Leggett, On the superfluid fraction of an arbitrary many-body system at T =0, J. Stat. Phys. 93, 927 (1998).

[12] I. Božović, X. He, J. Wu, and A. T. Bollinger, Dependence of the critical temperature in overdoped copper oxides on superfluid density, Nature (London) 536, 309 (2016).

[13] J. Zaanen, Superconducting electrons go missing, Nature (London) 536, 282 (2016).

[14] S. A. Kivelson, On the character of the superconductor to metal transition in overdoped cuprates, J. Club Condens. Matter Phys., https://doi.org/10.36471/JCCM_August_ 2016_01 (2016).

[15] F. Mahmood, X. He, I. Božović, and N. P. Armitage, Locating the Missing Superconducting Electrons in the Overdoped Cuprates $\mathrm{La}_{2-x} \mathrm{Sr}_{x} \mathrm{CuO}_{4}$, Phys. Rev. Lett. 122, 027003 (2019).

[16] N. R. Lee-Hone, J. S. Dodge, and D. M. Broun, Disorder and superfluid density in overdoped cuprate superconductors, Phys. Rev. B 96, 024501 (2017).

[17] N. R. Lee-Hone, V. Mishra, D. M. Broun, and P. J. Hirschfeld, Optical conductivity of overdoped cuprate superconductors: Application to $\mathrm{La}_{2-x} \mathrm{Sr}_{x} \mathrm{CuO}_{4}$, Phys. Rev. B 98, 054506 (2018).

[18] N. R. Lee-Hone, H. U. Özdemir, V. Mishra, D. M. Broun, and P. J. Hirschfeld, Low energy phenomenology of the overdoped cuprates: Viability of the Landau-BCS paradigm, Phys. Rev. Research 2, 013228 (2020).

[19] I. Božović, X. He, J. Wu, and A. T. Bollinger, The vanishing superfluid density in cuprates-and why it matters, J. Supercond. Novel Magn. 31, 2683 (2018).

[20] I. Božović, A. T. Bollinger, J. Wu, and X. He, Can high-T superconductivity in cuprates be explained by the conventional BCS theory?, Low Temp. Phys. 44, 519 (2018).

[21] D. T. Son, Low-energy quantum effective action for relativistic superfluids, arXiv:hep-ph/0204199.

[22] A. Nicolis, Low-energy effective field theory for finitetemperature relativistic superfluids, arXiv:1108.2513.

[23] L. V. Delacrétaz, D. M. Hofman, and G. Mathys, Superfluids as higher-form anomalies, Sci. Post. Phys. 8, 047 (2020).
[24] C. P. Herzog, P. K. Kovtun, and D. T. Son, Holographic model of superfluidity, Phys. Rev. D 79, 066002 (2009).

[25] See the Supplemental Material at http://link.aps.org/ supplemental/10.1103/PhysRevLett.124.161604 for contains details of the hydrodynamic theory, details of the holographic model, details on the holographic computation of the conductivity and the normal and superfluid densities as well as further comments on $\pi_{s}$ as a proxy for doping., which includes Refs. [10,26-34].

[26] M. A. Valle, Hydrodynamic fluctuations in relativistic superfluids, Phys. Rev. D 77, 025004 (2008).

[27] S. A. Hartnoll, C. P. Herzog, and G. T. Horowitz, Holographic superconductors, J. High Energy Phys. 12 (2008) 015 .

[28] T. Andrade and B. Withers, A simple holographic model of momentum relaxation, J. High Energy Phys. 05 (2014) 101.

[29] C. P. Herzog, N. Lisker, P. Surowka, and A. Yarom, Transport in holographic superfluids, J. High Energy Phys. 08 (2011) 052.

[30] P. K. Kovtun, D. T. Son, and A. O. Starinets, Viscosity in Strongly Interacting Quantum Field Theories from Black Hole Physics, Phys. Rev. Lett. 94, 111601 (2005).

[31] M. Blake, Universal Charge Diffusion and the Butterfly Effect in Holographic Theories, Phys. Rev. Lett. 117, 091601 (2016).

[32] S. A. Hartnoll, Theory of universal incoherent metallic transport, Nat. Phys. 11, 54 (2015).

[33] K. Skenderis, Lecture notes on holographic renormalization, Classical Quantum Gravity 19, 5849 (2002).

[34] R. A. Davison, B. Goutéraux, and S. A. Hartnoll, Incoherent transport in clean quantum critical metals, J. High Energy Phys. 10 (2015) 112.

[35] L. P. Kadanoff and P. C. Martin, Hydrodynamic equations and correlation functions, Ann. Phys. (N.Y.) 24, 419 (1963).

[36] P. Kovtun, Lectures on hydrodynamic fluctuations in relativistic theories, J. Phys. A 45, 473001 (2012).

[37] J. M. Maldacena, The Large N limit of superconformal field theories and supergravity, Int. J. Theor. Phys. 38, 1113 (1999); Adv. Theor. Math. Phys. 2, 231 (1998).

[38] E. Witten, Anti-de Sitter space and holography, Adv. Theor. Math. Phys. 2, 253 (1998).

[39] S. A. Hartnoll, A. Lucas, and S. Sachdev, Holographic quantum matter, arXiv:1612.07324.

[40] C. Charmousis, B. Goutéraux, B. S. Kim, E. Kiritsis, and R. Meyer, Effective Holographic Theories for low-temperature condensed matter systems, J. High Energy Phys. 11 (2010) 151.

[41] R. A. Davison, S. A. Gentle, and B. Goutéraux, Impact of irrelevant deformations on thermodynamics and transport in holographic quantum critical states, Phys. Rev. D 100, 086020 (2019).

[42] A. Adam, B. Crampton, J. Sonner, and B. Withers, Bosonic fractionalisation transitions, J. High Energy Phys. 01 (2013) 127.

[43] S. A. Hartnoll, C. P. Herzog, and G. T. Horowitz, Building a Holographic Superconductor, Phys. Rev. Lett. 101, 031601 (2008).

[44] C. P. Herzog and A. Yarom, Sound modes in holographic superfluids, Phys. Rev. D 80, 106002 (2009). 
[45] J. Sonner and B. Withers, A gravity derivation of the TiszaLandau model in AdS/CFT, Phys. Rev. D 82, 026001 (2010).

[46] J. Bhattacharya, S. Bhattacharyya, and S. Minwalla, Dissipative superfluid dynamics from gravity, J. High Energy Phys. 04 (2011) 125.

[47] J. Bhattacharya, S. Bhattacharyya, S. Minwalla, and A. Yarom, A Theory of first order dissipative superfluid dynamics, J. High Energy Phys. 05 (2014) 147.

[48] S. S. Gubser, Breaking an Abelian gauge symmetry near a black hole horizon, Phys. Rev. D 78, 065034 (2008).

[49] S. S. Gubser and A. Nellore, Ground states of holographic superconductors, Phys. Rev. D 80, 105007 (2009).

[50] G. T. Horowitz and M. M. Roberts, Zero temperature limit of holographic superconductors, J. High Energy Phys. 11 (2009) 015.

[51] B. Goutéraux and E. Kiritsis, Quantum critical lines in holographic phases with (un)broken symmetry, J. High Energy Phys. 04 (2013) 053.

[52] B. Goutéraux, Charge transport in holography with momentum dissipation, J. High Energy Phys. 04 (2014) 181.

[53] B. Goutéraux and E. Mefford, Temperature dependence of normal and superfluid densities in clean, quantum critical holographic superfluids (to be published).

[54] S. A. Hartnoll, Horizons, holography and condensed matter, in Black Holes in Higher Dimensions, edited by G. T. Horowitz (Cambridge University Press,, Cambridge, 2012), pp. 387-419.

[55] S. A. Hartnoll and L. Huijse, Fractionalization of holographic Fermi surfaces, Classical Quantum Gravity 29, 194001 (2012).

[56] A. Altland and B. D. Simons, Condensed Matter Field Theory, 2nd ed. (Cambridge University Press, Cambridge, England, 2010).

[57] A. Schmitt, Introduction to superfluidity, Lect. Notes Phys. 888, 1 (2015).

[58] The terms arise from the breaking of particle-hole symmetry and gauge invariance, respectively. Denoting the relative temperature dependence of these two terms by $T^{\alpha}$, we find a more general criteria $\alpha<d+2-\theta-z \Rightarrow \rho_{n}^{(0)}=0$. Here, we have $\alpha=0$. In different models [53] than the ones considered here, we can find fractionalized phases which have $\rho_{n}^{(0)}=0$, similar to ${ }^{4} \mathrm{He}$.

[59] M. Blake and A. Donos, Diffusion and chaos from near $\mathrm{AdS}_{2}$ horizons, J. High Energy Phys. 02 (2017) 013.

[60] R. A. Davison, S. A. Gentle, and B. Goutéraux, Slow Relaxation and Diffusion in Holographic Quantum Critical Phases, Phys. Rev. Lett. 123, 141601 (2019).

[61] A. Donos and J.P. Gauntlett, Holographic Q-lattices, J. High Energy Phys. 04 (2014) 040.

[62] Y. Ling, P. Liu, C. Niu, J.-P. Wu, and Z.-Y. Xian, Holographic superconductor on Q-lattice, J. High Energy Phys. 02 (2015) 059.
[63] T. Andrade and S. A. Gentle, Relaxed superconductors, J. High Energy Phys. 06 (2015) 140.

[64] K.-Y. Kim, K. Kiu Kim, and M. Park, A simple holographic superconductor with momentum relaxation, J. High Energy Phys. 04 (2015) 152.

[65] L. D. Landau and E. M. Lifshitz, Fluid Mechanics (Second Edition), edited by L. D. Landau and E. M. Lifshitz, 2nd ed. (Pergamon, 1987).

[66] A. Yarom, Fourth sound of holographic superfluids, J. High Energy Phys. 07 (2009) 070.

[67] R. A. Davison, L. V. Delacrétaz, B. Goutéraux, and S. A. Hartnoll, Hydrodynamic theory of quantum fluctuating superconductivity, Phys. Rev. B 94, 054502 (2016); Erratum, Phys. Rev. B 96, 059902 (2017).

[68] Y. Wang, J. Yan, L. Shan, H.-H. Wen, Y. Tanabe, T. Adachi, and Y. Koike, Weak-coupling $d$-wave bcs superconductivity and unpaired electrons in overdoped $\mathrm{la}_{2-x} \mathrm{Sr}_{x} \mathrm{CuO}_{4}$ single crystals, Phys. Rev. B 76, 064512 (2007).

[69] H.-H. Wen, Z.-Y. Liu, F. Zhou, J. Xiong, W. Ti, T. Xiang, S. Komiya, X. Sun, and Y. Ando, Electronic specific heat and low-energy quasiparticle excitations in the superconducting state of $\mathrm{La}_{2-x} \mathrm{Sr}_{x} \mathrm{CuO}_{4}$ single crystals, Phys. Rev. B 70, 214505 (2004).

[70] In using $\phi_{s} / \mu$ as a proxy for doping, we note that its variation triggers a quantum phase transition between a superfluid and normal phase. Similarly to doping, increasing $\phi_{s} / \mu$ increases the total charge density, $\rho_{T}^{(0)}$, as well as amplifies the effect of momentum breaking (increased disorder strength) [25].

[71] N. E. Hussey, R. A. Cooper, X. Xu, Y. Wang, B. Vignolle, and C. Proust, Dichotomy in the $T$-linear resistivity in holedoped cuprates, arXiv:0912.2001.

[72] S. Badoux, W. Tabis, F. Laliberté, G. Grissonnanche, B. Vignolle, D. Vignolles, J. Béard, D. A. Bonn, W. N. Hardy, R. Liang, N. Doiron-Leyraud, Louis Taillefer, and Cyril Proust, Change of carrier density at the pseudogap critical point of a cuprate superconductor, Nature (London) 531, 210 (2016).

[73] C. Putzke, S. Benhabib, W. Tabis, J. Ayres, Z. Wang, L. Malone, S. Licciardello, J. Lu, T. Kondo, T. Takeuchi, N. E. Hussey, J. R. Cooper, and A. Carrington, Reduced Hall carrier density in the overdoped strange metal regime of cuprate superconductors, arXiv:1909.08102.

[74] A. Abanov, A. V. Chubukov, and J. Schmalian, Quantumcritical theory of the spin-fermion model and its application to cuprates: Normal state analysis, Adv. Phys. 52, 119 (2003).

[75] M. A. Metlitski and S. Sachdev, Quantum phase transitions of metals in two spatial dimensions: II. Spin density wave order, Phys. Rev. B 82, 075128 (2010).

[76] A. A. Patel and S. Sachdev, DC resistivity at the onset of spin density wave order in two-dimensional metals, Phys. Rev. B 90, 165146 (2014). 